\title{
Herpesvirus associé à la maladie de Kaposi KSHV/HHV8: nouveaux résultats et nouvelles questions
}

médecine/sciences $\left(n^{\circ} 6\right.$, vol. $11, p$. 914) a récemment rapporté la découverte d'un "nouveau" virus humain de la famille des herpesvirus, identifié par une technique d'amplification différentielle de fragments d'ADN dans des lésions de maladie de Kaposi associée au SIDA [1]. Ce virus est actuellement désigné KSHV pour Kaposi's sarcoma-associated herpesvirus, en attendant une nomenclature officielle; il est parfois surnommé HHV8. L'identification d'un nouvel herpesvirus humain potentiellement transformant, associé, notamment, à une maladie très fréquente au cours du SIDA, a d'emblée suscité un large intérêt et la mise en route de nombreux travaux. Plusieurs résultats ont été récemment publiés ou communiqués de façon préliminaire.

\section{Le virus KSHV}

Les données concernant le virus KSHV sont encore limitées. Seules les séquences des deux courts fragments d'ADN de l'article initial de ('hang et al. sont actuellement publiées [1]. Le clonage de deux grands fragments de $12 \mathrm{~kb}$ et $21 \mathrm{~kb}$ permettant l'identification d'une vingtaine de cadres ouverts de lecture a été rapporté de façon préliminaire [2]. Ces fragments partagent des homologies de séquences et une colinéarité avec l'herpesvirus saimiri (HVS, primate) et dans une moindre mesure avec le virus d'Epstein-Barr (EBV, humain), les deux membres connus de la sousfamille des $\gamma$-Herpes viridae [3]. De façon très intéressante, certaines protéines potentielles du KSHV sont proches de protéines de mammifère, en particulier des cyclines D et des récepteurs de membrane couplés $\mathrm{m} / \mathrm{s} n^{\circ} 11$, vol. 11, novembre 95 aux protéines $G$. Des produits viraux analogues semblent impliqués dans les leucémies ou lymphomes induits par l'HVS chez certains primates. Plusieurs lignées lymphoblastoïdes ou lymphomateuses infectées sont disponibles, contenant chacune de nombreuses copies du génome viral. Celui-ci apparaît en structure épisomique, localisé dans le noyau, migrant sur gel à une taille d'environ $270 \mathrm{~kb}$ (contre $112 \mathrm{~kb}$ pour l'HVS et $172 \mathrm{~kb}$ pour l'EBV). Le virus KSHV entier n'a pas encore été isolé. Il n'y a pas actuellement d'anticorps disponibles permettant d'évaluer la séroprévalence de l'infection.

\section{KSHV et pathologie}

\section{- Maladie de Kaposi}

Plusieurs études (portant sur un total de plus de 200 cas) ont confirmé la présence de séquences KSHV détectées par PCR dans plus de $95 \%$ des tissus de maladie de Kaposi, dans toutes ses formes clinico-épidémiologiques: classique (atteinte sporadique de sujets âgés originaires du pourtour méditerranéen et juifs d'Europe centrale), endémique (sujets jeunes d'Afrique équatoriale), iatrogène (liée à des traitements immunosuppresseurs), épidémique (associée à l'infection par le VIH), et enfin chez des patients homosexuels non infectés par le VIH [1-2, 4-8]. Les séquences virales ont également été recherchées par Southern blot, et sont détectables dans la majorité des cas positifs en PCR par cette technique moins sensible et donc à moindre risque de contamination [ 1 , 2]. L'intensité des signaux en Southern blol permet de prédire un nombre moyen dans un échantillon de une ou deux copies d'ADN viral par cellule [1]. Des résultats préliminaires d'hybridation in situ localiseraient les séquences virales dans les cellules fusiformes typiques de la maladie, d'origine probablement vasculaire [2]. D'autres tissus de sujets atteints de maladie de Kaposi ont été testés par PCR: les séquences KSHV sont parfois détectées dans des échantillons de peau saine juxtalésionnelle, dans les cellules sanguines mono-nucléées (notamment dans la fraction $\mathrm{CD}_{19^{+}}$, c'est-à-dire lymphoïde B), ou dans le sperme, mais non dans la salive $[2,7,8]$. De nombreux témoins négatifs ont été effectués sur des tissus de sujets indemnes de maladie de Kaposi, infectés ou non par le VIH. Les séquences virales n'ont pas été détectées dans ces prélèvements, mis à part dans de rares ganglions hyperplasiques ou lymphomateux ([1], et voir ci-dessous).

La forte association entre la maladie de Kaposi (de tous types) et la détection des séquences virales étant établie, le virus KSHV est fortement soupçonné d'être l'agent responsable de cette maladie. Cette relation causale a pourtant été mise en doute, notamment par R. Gallo, spécialiste des herpesvirus et de la maladie de Kaposi. Les objections, indirectes, sont les suivantes: (1) les séquences virales deviennent rapidement indétectables dans les cultures in vitro de tissus de maladie de Kaposi, et la plupart des lignées établies ne contiennent pas ces séquences $[2,7,8]$. Par conséquent, soit le virus est présent dans des cellules associées ne proliférant pas en culture, soit les cellules initialement infectées perdent in vitro le génome viral épisomique; (2) la 
spécificité de l'association entre le virus KSHV et la maladie de Kaposi a été remise en question : les séquences virales ont été détectées par PCR dans 27 des 33 tumeurs cutanées variées étudiées (kératose actinique, carcinomes...) survenues chez quatre sujets transplantés traités par immunosuppresseurs, non infectés par le VIH, en dehors de tout contexte de maladic de Kaposi [9]. L'hvpothèse des auteurs est que le virus pourrait être présent dans l'organisme à l'état latent et se développer dans les tissus en prolifération chez les sujets immunodéprimés. Il n'est pas exclu que, de façon similaire, le virus ne soit qu'un "passager " clans les lésions de maladie de Kaposi; (3) les caractéristiques épidémiologiques habituelles des herpesvirus ne sont pas celles attendues pour "l'agent de la maladie de Kiposi ". Parmi les sujets infectés par le VIH, les homosexuels développent bien plus souvent une maladie de Kaposi que les hémophiles ou les toxicomanes, ce qui a fait soupçonner l'existence d'un agent de la maladie de Kaposi transmissible par voie sexuelle, de portage restreint à cette population à risque. (Or les herpesvirus sont habituellement largement répandus dans la population où ils persistent che\% les individus à l'état latent, avec parfois des épisodes de réactivation.

\section{- KSHV et body-cavity-based lymphoma}

La parenté du KSHV avec les virus HV'S et EBV', deux virus lymphotropes impliqués dans la survenue de lymphomes, ainsi que l'association non aléatoire entre la maladie de Kaposi et les lymphomes chez les sujets infectés ou non par le VIH, ont amené à rechercher les séquences virales dans la pathologie lymphomateuse. Plus de 200 lymphomes de tous types associés ou non au SIDA ont été testés par les auteurs à l'origine de la mise en évidence des séquences KSHV [2, 10]. Seul le sousgroupe très particulier des body-carilybased lymphomas, "lymphomes des cavités séreuses de l'organisme", est associé de façon significative aux séquences virales. Il s'agit d'une forme rare de lvmphome le plus souvent associé au SII)A, constituće d'épan- chements lymphomateux primitifs des cavités pleurale, péricardique et/ou péritonéale, sans masse tumorale. Les cellules lymphomateuses, de type $B$, contiennent toutes des séquences $\mathrm{EBV}$ et, soit de nombreuses séquences KSHV (40 à 80 copies par cellule), soit, plus rarement, un réarrangement de l'oncogène $c-M Y(;$; suggérant deux mécanismes alternatifs de transformation $[2,10]$. La co-infection par les virus EBV et KSHV joue probablement un rôle dans l'oncogenèse de cette prolifération lymphoïde $B$.

Par ailleurs, le groupe de Biberfeld associé à celui de (aallo a rapporté de façon préliminaire la détection des séquences KSHV par PCR dans une série de onze cas (dont dix non associés au VIH) de lvmphomes africains de types variés [2].

\section{- KSHV et maladie de Castleman multicentrique}

Il s'agit d'une maladie rare, se présentant typiquement par une hyperplasie disséminée ganglionnaire et splénique, constituée d'une prolifération cellulaire polyclonale plasmocytaire et plus ou moins hyalino-vasculaire [11]. Une sécrétion anormale d'interleukine 6 apparaît responsable de la prolifération lymphoïde et des signes systémiques souvent présents. Des formes associées à l'infection par le VIH sont décrites. Les séquences KSHV ont été recherchées dans cette maladie en raison de son association à la maladie de Kiposi (dans $13 \%$ des cas, et dans plus de $75 \%$ des cas positifs pour le VIH), et de caractéristiques communes aux deux proliférations. Les séquences virales ont été détectées par PCR dans $7 / 17$ biopsies ganglionnaires de patients non infectés par le VIH avec, estimé en Southern blot, un nombre variable de copies virales par échantillon ([12] et résultats non publiés). Par comparaison, ces séquences n'ont été retrouvées par PCR que dans $1 / 35$ hyperplasies folliculaires et/ou interfolliculaires non spécifiques, négatives pour le VIH. Chez les patients infectés par le VIH et atteints de maladie de Castleman multicentrique isolée ou associée à une maladie de Kaposi, les séquences
KSHV ont été détectées par PCR et Southern blot dans tous les cas [12]. Il faudra déterminer si le virus KSHV joue un rôle dans la prolifération cellulaire de ces cas de maladie de Castleman.

Par ailleurs, quelques cas de lymphadénopathie angio-immunoblastique et d'hyperplasie folliculaire, positifs en PCR pour le KSHV, ont été rapportés de façon préliminaire par une équipe italienne [2].

L'identification du virus KSHV par Chang et Moore a ouvert de nombreuses perspectives. $\mathrm{Si}$ son existence même ne semble plus faire de doute, il reste à isoler et caractériser ce virus. Il faudra déterminer la fréquence de l'infection chez l'homme, sa répartition géographique, ses modes de transmission, les cellules contenant le virus chez les sujets infectés et notamment dans la maladie de Kaposi, l'existence de protéines virales transformantes et leur mode d'action. Les connaissances lentement accumulées sur l'EBV ont montré qu'un même virus pouvait, à la fois, être présent chez la majorité de la population à l'état latent et jouer un rôle dans la survenue d'une maladie bénigne (la mononucléose infectieuse), de cancers (cancer du nasopharynx, lymphome de Burkitt endémique, certains lymphomes associés ou non au SIDA), et de proliférations lymphoïdes survenant après transplantation. De même, le spectre pathologique du virus KSHV pourrait être varié. Bien qu'il existe une forte association entre ce virus et la maladie de Kaposi, les body cavitybased lymphoma, et la maladie de Castleman (surtout chez les patients positifs pour le VIH), les données actuelles ne permettent pas d'affirmer ou de récuser sa responsabilité dans ces affections.

J.S. 
1. Chang Y, Cesarman E, Pessin MS, et al. Identification of Herpesvirus-like DNA sequences in AIDS-associated Kaposi's sarcoma. Science 1994; 266: 1865-9.

2. Abstracts for the 1995 annual meeting sponsored by the laboratory of tumor cell biology, NC.I. AIDS Res Hum Retrovir 1995; I I, suppl 1.

3. Albrecht JC, Nicholas J, Biller D, et al. Primary structure of the herpesvirus saimiri genome. J Virol 1992 ; 66 : 5047-58.

4. Moore PS, Chang Y. Detection of herpesviruslike DNA sequences in Kaposi's sarcoma in patients with and those without HIV infection. $N$ Engl J Med 1995; 332: 1 181-5.

5. Huang YQ Li JJ, Kaplan MH, et al. Herpesviruslike nucleic acid in various forms of Kaposi's sarcoma. Lancet $1995 ; 345$ : 759-61

6. Dupin N, Grandadam M, Calvez V, et al. Herpesvirus-like DNA sequences in patients with Mediterranean Kaposi's sarcoma. Lancet 1995; 345: $761-2$

7. Ambroziak JA, Blackbourn DJ, Heindier BG, et al. Herpes-like sequences in HIV infected and uninfected Kaposi's sarcoma patients (technical comment). Science 1995; 268: 582-3.

8. Lebbé C., de Crémoux P, Rybojad M, et al. Kaposi's sarcoma and new herpes virus (letter) Lancet $1995 ; 345$ : 1180.

9. Rady PL, Yen A, Rollef son JL, et al. Herpesviruslike DNA sequences in nonKaposi's sarcoma skin lesions of transplant patients. Lancet 1995; 345: 1330-4.

10. Cesarman E, Chang Y, Moore PS, Said JW, Knowles DM. Kaposi's sarcoma associated herpes virus like DNA sequences in AIDS related body cavity based lymphomas. N EnglJ Med 1995; 332 : $1186-91$.

11. Peterson BA, Frizzera G. Multicentric Castleman's disease. Semin Oncol 1993; 20 : 636.47.

12. Soulier J, Grollet L, Oksenhendler E, et al. Kaposi's sarcoma associated Herpesvirus-like DNA sequences in multicentric Castleman's disease. Blood 1995; 86: 1276-80.

\section{BRÈVES}

Les enhancers augmentent la probabilité qu'un gène soit transcrit, non le taux de transcription. L'action stimulatrice d'une séquence enhancer sur un promoteur hétérologue est retrouvée in vitro et mise en évidence par l'augmentation du nombre de transcrits. Cette constatation ne résout cependant pas la question du mécanisme en cause: augmentation du taux de transcription dans chaque noyau transfecté, ou augmentation du nombre de cellules exprimant le transcrit. Un groupe américain de Seattle vient d'apporter à cette question une réponse élégante par l'utilisation simultanée de deux séries de techniques [1]. Un gène rapporteur, codant pour la $\beta$-galactosidase $(\beta-G a l)$, a été lié à un promoteur de thymidine kinase du virus de l'herpes (HSV-tk LacZ) et transfecté dans des cellules HeLa, avec ou sans addition d'un enhancer SV40 en aval. Au bout de soixantedouze heures, la culture a subi une coloration par fluorescence ; un tri cellulaire (FACS) analysant l'expression de $\beta$-Gal a mis alors en évidence l'augmentation d'environ 10 fois du nombre de cellules exprimant ce gène. Cette augmentation est parallèle à l'augmentation constatée de la transcription, l'action du enhancer étant une action "tout ou rien". Ces résultats ont été corroborés par les expériences en transfection stable. L'expérience conforte donc le modèle probabiliste par opposition au modèle quantitatif (figure 1). On peut supposer que le enhancer agit au niveau de la chromatine en favorisant la formation d'une structure active stable; le taux de transcription variable serait alors sous le contrôle d'autres éléments situés en cis et faisant partie du contexte chromosomique.

[1. Walters MC, et al. Proc Natl Acad Sci USA 1995 ; 92 : 7125-9.]

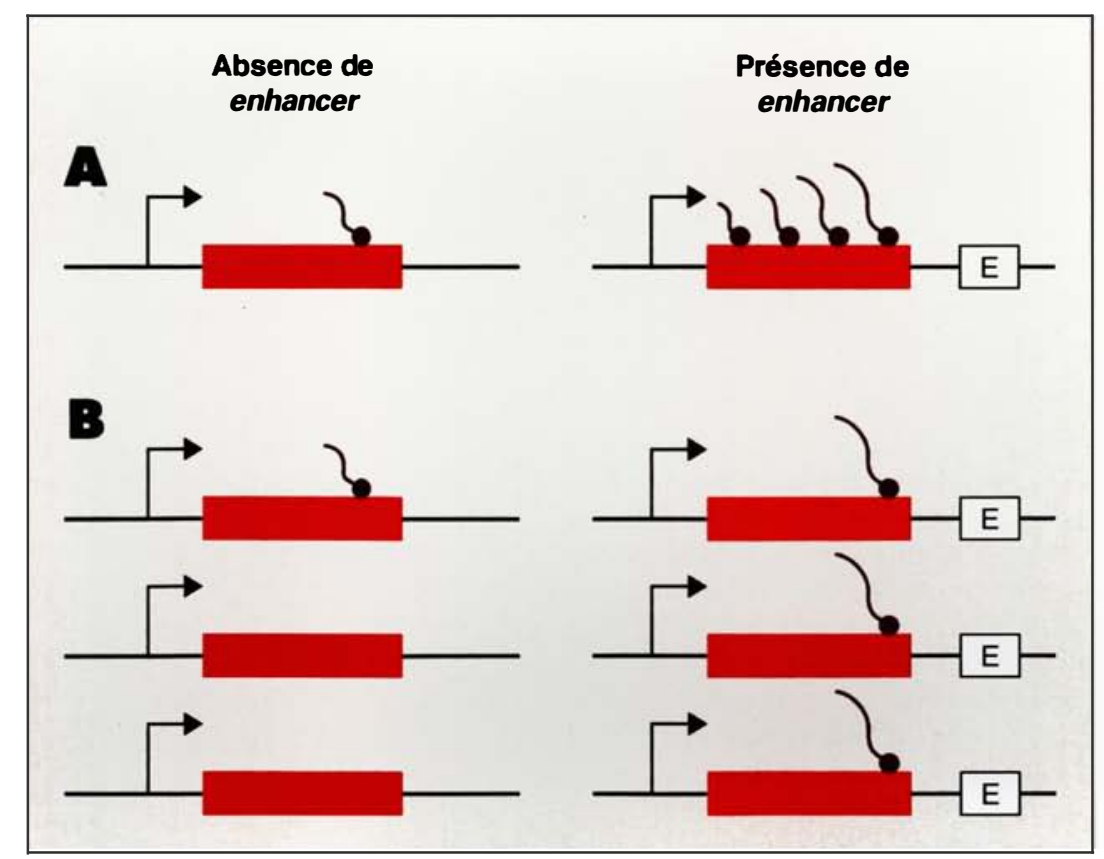

Figure 1. Représentation schématique des deux modèles, quantitatif et probabiliste. A. Dans le modèle quantitatif, la présence d'un enhancer augmente la densité de la polymérase au niveau de l'unité de transcription. $B$. Dans le modèle probabiliste, le enhancer augmente le nombre de matrices recrutées, sans qu'il y ait augmentation de la densité de la polymérase. 